\title{
Atresia duodenal con apple peel asociada a hernia diafragmática congénita: un caso excepcional y revisión de la literatura
}

\author{
J.A. Molino, S. López Fernández, B. Oliver, H. Boix, O. Rocha, M. López, G. Guillén
}

Servicio de Cirugía Pediátrica. Unidad de Cirugía Oncológica y Neonatal. Hospital Universitario Vall d’Hebron. Barcelona.

\begin{abstract}
RESUMEN
Introducción. La atresia duodenal asociada con appel peel es extremadamente infrecuente. La primera se produce por un defecto en la recanalización en etapas tempranas, mientras que la atresia intestinal parece deberse a causas vasculares en etapas más tardías. La presencia de anomalías asociadas a la hernia diafragmática es común, pero la asociación con la atresia duodenal apenas está descrita.

Caso clínico. Presentamos un recién nacido de 31 semanas de gestación y sexo femenino, con atresia duodenal y apple peel, asociada a hernia diafragmática izquierda y cardiopatía mayor. Se realizó un flap muscular abdominal para el cierre del defecto diafragmático y anastomosis duodenoyeyunal tras la resección de parte del apple peel inviable.

Comentarios. A nuestro entender, es el primer caso descrito con esta asociación singular. La combinación de atresia duodenal con apple peel se ha descrito previamente en 11 ocasiones; la asociación de ambas con hernia diafragmática congénita no había sido descrita.
\end{abstract}

Palabras Clave: Atresia duodenal; Atresia intestinal; Apple peel; Hernia diafragmática congénita; Malformación congénita.

\section{DUODENAL ATRESIA WITH APPLE PEEL ASSOCIATED WITH CONGENITAL DIAPHRAGMATIC HERNIA: AN EXCEPTIONAL CASE AND A LITERATURE REVIEW}

\begin{abstract}
Introduction. Duodenal atresia associated with apple peel is extremely rare. Duodenal atresia occurs as a result of absence of recanalization at an early stage, whereas intestinal atresia is seemingly due to vascular causes at later stages. The presence of abnormalities associated with diaphragmatic hernia is frequent, but association with duodenal atresia has been little explored.

Care report. This is the case of a female neonate born at gestational week 31, with duodenal atresia and apple peel, associated with left diaphragmatic hernia and major heart disease. An abdominal mus-
\end{abstract}

DOI: $10.54847 /$ cp.2022.01.06

Correspondencia: Correspondencia: Dr. José Andrés Molino Gahete.

E-mail: jamolino@vhebron.net

Recibido: Noviembre 2020

Aceptado: Mayo 2021 cle flap was performed for diaphragmatic defect closure purposes, and duodenojejunal anastomosis was carried out following resection of part of the non-viable apple peel.

Discussion. To our knowledge, this is the first case described with this rare association. The combination of duodenal atresia and apple peel had been previously described 11 times. However, the association of both with congenital diaphragmatic hernia had not been reported yet.

KeY Words: Duodenal atresia; Intestinal atresia; Apple peel; Congenital diaphragmatic hernia; Congenital malformation.

\section{INTRODUCCIÓN}

La atresia duodenal es la causa congénita más común de obstrucción intestinal neonatal, su incidencia es de una por cada 5.000-10.000 recién nacidos, representa la mitad de las atresias intestinales ${ }^{(1)}$. Una de las teorías más aceptadas para su formación es un defecto en la recanalización a partir de la $6^{\mathrm{a}}$ semana de gestación $(\mathrm{SG})^{(2)}$.

La atresia intestinal, en cambio, se produce por la interrupción del aporte vascular en etapas más tardías de la gestación ${ }^{(3,4)}$. La atresia intestinal tipo IIIb, denominada apple peel, representa aproximadamente un 5\% de las atresias yeyunoileales $^{(1)}$. La asociación entre atresia duodenal y atresia intestinal apple peel es excepcional y hasta el momento solo se han descrito 11 casos en la literatura.

La hernia diafragmática congénita se da en uno de cada 2.500 a 3.500 recién nacidos. La asociación con otras malformaciones es común, siendo las cardiacas las más frecuentes, mientras que la asociación con malformaciones gastrointestinales no es habitual ${ }^{(5)}$. La asociación de hernia diafragmática congénita con atresia duodenal apenas está descrita ${ }^{(6)}$.

La asociación de las tres malformaciones no tiene una explicación embriológica satisfactoria que las englobe en su patogénesis, por lo que podríamos pensar en una causa genética. Se ha descrito la asociación de atresia intestinal familiar, principalmente apple peel, con el receptor 2IIIb y 10 del factor de crecimiento de fibroblastos (Fgfr2b/Fgf10) 


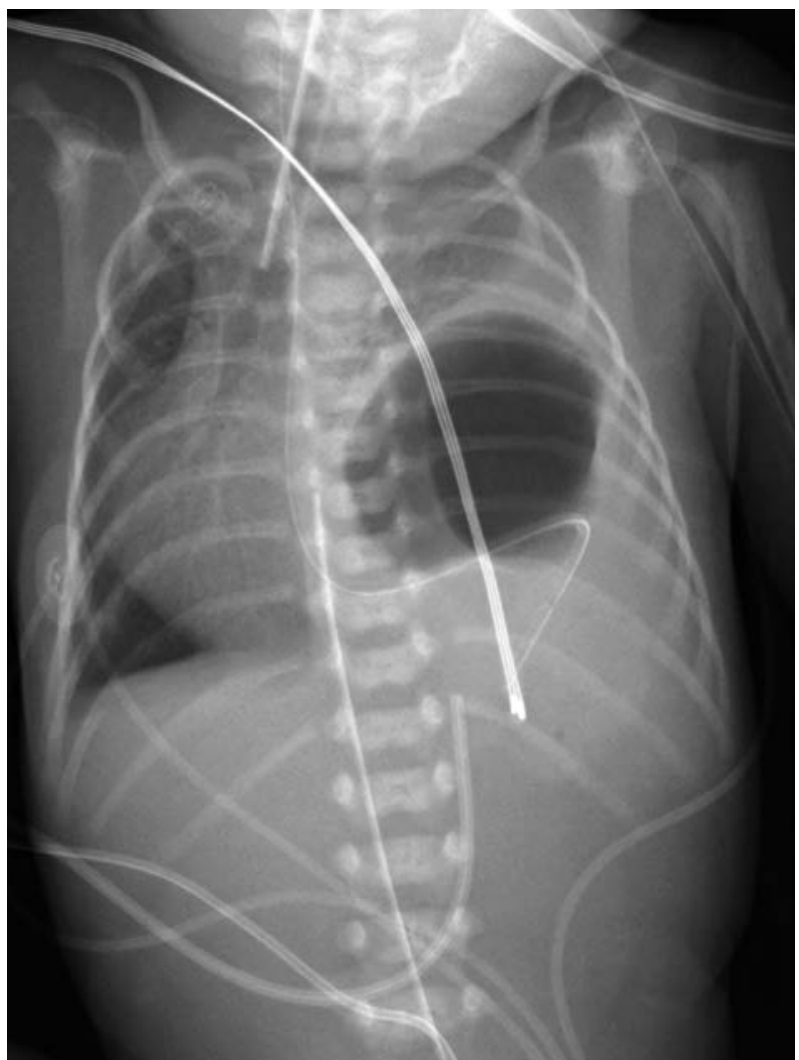

Figura 1. Radiografía toracoabdominal al nacimiento: hernia diafragmática congénita izquierda, gran burbuja aérea en hemitórax izquierdo y sin aireación abdominal.

asociación de apple peel familiar y cardiopatía con mutaciones en el gen $\mathrm{NOTCH}^{(8)}$.

Presentamos un recién nacido pretérmino, con hernia diafragmática congénita y cardiopatía mayor asociada a atresia duodenal con apple peel.

\section{CASO CLÍNICO}

Gestante de 29 años con gestación gemelar monocorial biamniótica. El primer gemelo, de sexo femenino, presentaba polihidramnios y diagnóstico prenatal de hernia diafragmática izquierda con probable herniación de intestino delgado, estómago e hígado (Lung-to-Head Ratio [LHR] 1,7 y o/e LHR $40 \%$ ). Además, presentaba una imagen ecogénica de un asa intestinal dilatada en tórax, sugestiva de obstrucción intestinal alta en la ecografía del segundo trimestre.

A las $31+4$ semanas de gestación se realiza cesárea por dinámica uterina de una neonata de 1.300 gramos y Apgar $5 / 7 / 7$. En la radiografía inicial se confirma la presencia de hernia diafragmática izquierda y dilatación gástrica sin presencia de aireación abdominal (Fig. 1). Además, se confirma el diagnóstico prenatal de cardiopatía consistente en tetralogía de Fallot con doble salida del ventrículo derecho.

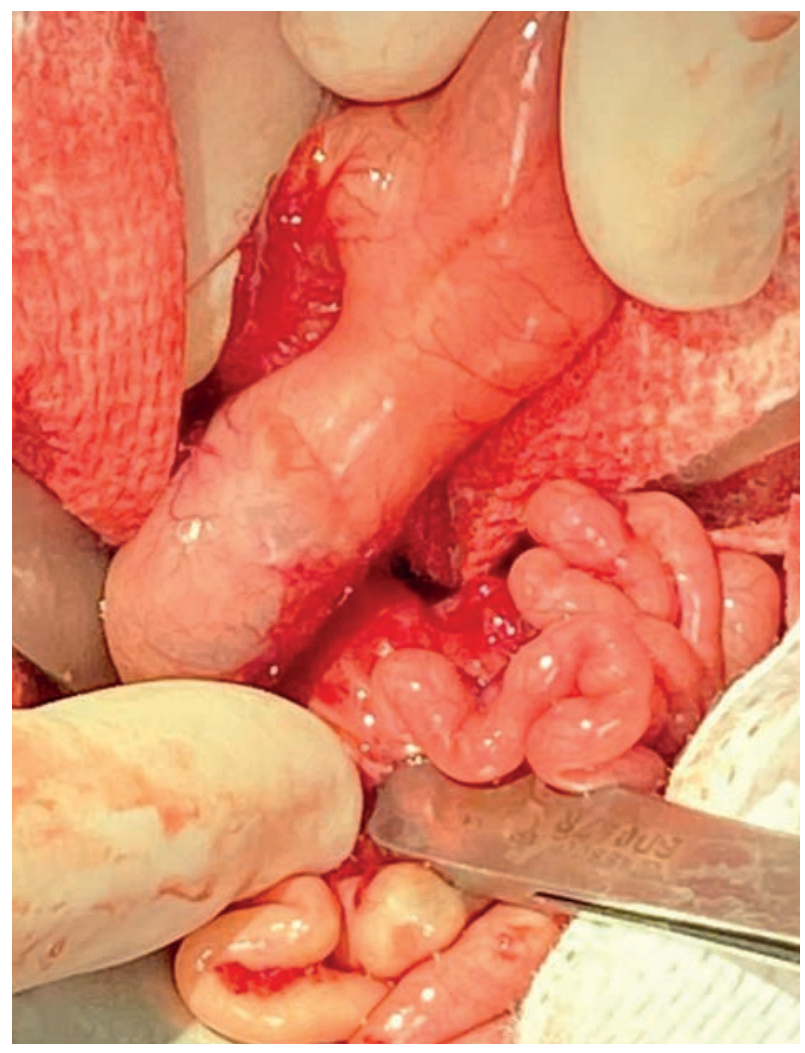

Figura 2. Atresia duodenal tipo III postampular.

Es intervenida el tercer día de vida, tras ser estabilizada, por laparotomía transversa supraumbilical izquierda, hallando agenesia diafragmática izquierda casi en su totalidad que requiere la confección de un flap muscular abdominal con oblicuo menor y transverso para el cierre del defecto, como técnica de elección empleada en nuestro centro y descrita previamente ${ }^{(9)}$.

Tras reducir las vísceras herniadas se aprecia gran dilatación gástrica con atresia duodenal postampular sin continuidad con el resto de intestino (Fig. 2), a nivel yeyunal presenta conformación tipo appel peel. Tras resecar los primeros 50 $\mathrm{cm}$ de apple peel inviables, se realizó anastomosis duodenoyeyunal, preservando $85 \mathrm{~cm}$ de intestino delgado hasta válvula ileocecal (Fig. 3).

Se mantiene intubada 10 días, tanto por la hipoplasia pulmonar como por su cardiopatía de base, y con nutrición parenteral total o parcial hasta los 19 días de vida, cuando se consigue la nutrición enteral completa (34 SG corregidas). Es dada de alta a los 115 días con un peso de $3,430 \mathrm{~kg}$.

Se realizó estudio genético que confirmaba que se trataba de gemelas monocigotas, sin mostrar alteraciones genéticas remarcables de la paciente con respecto a su hermana.

En un segundo ingreso, se intervino de la cardiopatía por presentar crisis hipóxicas graves atribuidas principalmente a su cardiopatía y no tanto a la hipoplasia pulmonar. Se realizó mediante la creación de una fístula sistémico-pulmonar, dilata- 


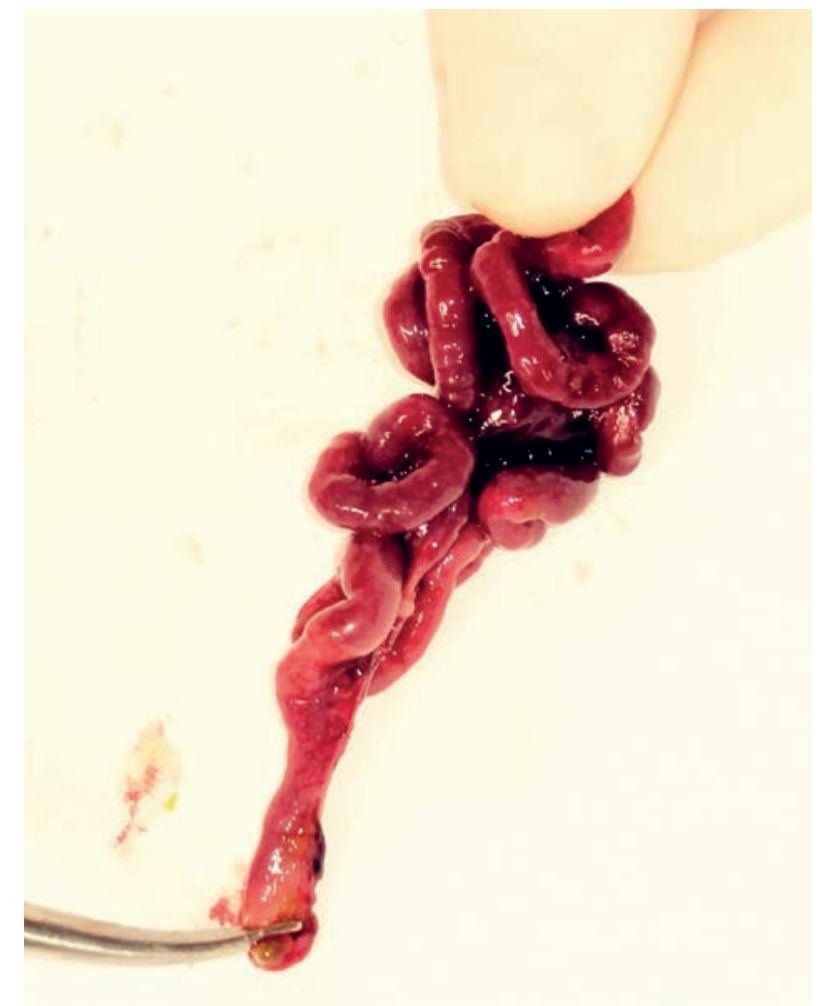

Figura 3. Segmento de apple peel resecado (yeyuno proximal).

ción de ambas venas pulmonares y cierre del ductus arterioso, requiriendo soporte postoperatorio en ECMO (oxigenación por membrana extracorpórea) durante cuatro días.

Actualmente, la paciente tiene 10 meses de vida, presenta un desarrollo ponderoestatural adecuado, con una correcta tolerancia a la dieta enteral, alternando nutrición oral con sonda nasogástrica por presentar trastornos de la succión-deglución.

\section{DISCUSIÓN}

La hernia diafragmática congénita es una malformación relativamente común, que se da en uno de cada 2.500 a 3.500 recién nacidos. Como en el caso descrito, la mayoría son posterolaterales izquierdas y el tamaño del defecto puede variar desde pequeños orificios herniarios hasta agenesias diafragmáticas, estando relacionado el tamaño del defecto con el tipo de reparación y el grado de hipoplasia pulmonar y, por tanto, con el pronóstico.

La etiología no está clara pero generalmente se considera esporádica, aunque hay casos hereditarios y asociados a síndromes y cromosomopatía. Su asociación con otras malformaciones es habitual, entre el 20 y $60 \%$ de los casos según las series, siendo la cardiopatía la malformación asociada más frecuente, seguida de las genitourinarias y musculoesqueléticas. La asociación con malformaciones gastrointestinales no es común, estando presente en menos del 5\% de los $\operatorname{casos}^{(5)}$.
En la serie publicada por Zaiss y cols., las malformaciones asociadas más frecuentes son las cardiovasculares y ocurren en un $50,4 \%$ de los pacientes. En esta serie las malformaciones gastrointestinales son el segundo grupo más frecuente en contraposición a lo publicado anteriormente y están presentes en un $21 \%$ de los pacientes y representan un $11,4 \%$ del total de las malformaciones asociadas divididas por aparatos. Dentro de las malformaciones gastrointestinales, el divertículo de Meckel y la atresia anal son las más frecuentes ${ }^{(10)}$. Ni en esta serie ni en la anterior se describe la asociación con atresia duodenal $^{(5)}$.

Castle y cols. describen un paciente con asociación VACTERL-like, compuesta por hemivértebra, clinodactilia, ano imperforado, atresia duodenal y hernia diafragmática derecha con fusión hepatopulmonar. Es la primera vez que se describe un caso similar y hacen referencia de forma excepcional a otros neonatos con hernia diafragmática y atresia duodenal( ${ }^{(6)}$.

La atresia duodenal tiene una incidencia de uno por cada 5.000-10.000 recién nacidos, es la causa más común de obstrucción congénita neonatal y, en la mayoría de los casos, la obstrucción es postampular. Aunque actualmente tiene una supervivencia por encima del 90\%, la prematuridad o la presencia de anomalías mayores asociadas, principalmente las cardiacas o cromosomopatías, pueden ensombrecer el pronóstico. Puede asociarse a páncreas anular o malrotación intestinal hasta en un 30\%, pero la asociación con atresias intestinales yeyunoileales se da en menos de un 5\% de los casos ${ }^{(1)}$.

$\mathrm{La}$ atresia intestinal tiene una incidencia de $0,7-0,8$ por cada 10.000 recién nacidos. Un 5\% de las atresias yeyunoileales son las denominadas apple peel, que consisten en una atresia proximal, a nivel yeyunal generalmente, mientras que el intestino delgado distal está enrollado a modo de espiral alrededor del vaso mesentérico nutricio. La importancia de este tipo de atresias está en que pueden requerir cirugías más complejas y dar lugar al síndrome de intestino corto $^{(3,4)}$.

Una de las explicaciones más aceptadas para la difícil asociación entre atresia duodenal e intestinal radica en sus diferentes etiologías embriológicas. En el caso de las atresias duodenales, una de la teorías más aceptadas es la falta de recanalización duodenal a partir de la $6^{\mathrm{a}} \mathrm{SG}^{(2)}$; en cambio, el resto de las atresias intestinales son consecuencia de una alteración en el aporte vascular en etapas más tardías ${ }^{(3,4)}$. En el caso del duodeno, el doble aporte vascular, tanto de la arteria mesentérica superior como del tronco celíaco a través de la arteria gastroduodenal, hacen más difícil la aparición de atresia duodenal por causa vascular ${ }^{(11)}$.

Por todo esto, la asociación entre atresia duodenal y atresia intestinal no es frecuente, siendo la asociación con atresia intestinal tipo apple peel extremadamente rara. Hasta el momento solo se han publicado 11 casos en la literatura científica $^{(7,11-20)}$, siendo nuestro paciente el número 12 y el único en asociarse además con hernia diafragmática congénita (Tabla I).

Además de la etiología embriológica se han descrito causas genéticas en el origen de las atresias intestinales, tanto de 
Tabla I. Atresia duodenal con apple peel. Revisión de la literatura.

\begin{tabular}{cllll} 
Caso & Autor & Año & SG & Anomalías asociadas \\
\hline 1 & Weber y cols. & 1999 & 36 & Agenesia AMS. Síndrome de Down. CIV \\
\hline 2 & Arbell y cols. & 2006 & 32 & Quiste de colédoco. Malrotación \\
\hline 3 & Tatekawa y cols. & 2007 & 36 & Atresia intestinal múltiple \\
\hline 4 & Ahman y cols. & 2009 & 34 & Agenesia de AMS \\
\hline 5 & Patil y cols. & 2011 & 33 & Malrotación \\
\hline 6 & Altokhais y cols. & 2014 & 33 & Agenesia de AMS. Malrotación. Microoftalmia \\
\hline 7 & Alnosair y cols. & 2014 & 31 & Agenesia de AMS \\
\hline 8 & Pathak y cols. & 2014 & 33 & \\
\hline 9 & Sasa y cols. & 2016 & 29 & Agenesia de AMS \\
\hline 10 & Ben Hamida y cols. & 2016 & 34 & Agenesia de AMS. Situs inverso abdominal. Atresia intestinal múltiple \\
\hline 11 & Kirtane y cols. & 2019 & 36 & CIA \\
\hline 12 & Molino y cols. & 2020 & 31 & HDC izquierda. Tetralogía de Fallot con doble salida VD \\
SG: semanas de gestación; AMS: arteria mesentérica superior; CIV: comunicación interventricular; CIA: comunicación interauricular; HDC: hernia diafragmática \\
congénita; VD: ventrículo derecho.
\end{tabular}

atresias intestinal tipo apple peel como de atresia duodenal familiar. La combinación de ambas atresias es extremadamente rara, del mismo modo que la existencia de atresias familiares como resultado de anomalías genéticas hereditarias. Los receptores 2IIIb y 10 del factor de crecimiento de fibroblastos (Fgfr2b y Fgf10) son moléculas reguladoras relevantes para las interacciones mesenquimatosa-epiteliales y están implicados en la patogénesis de la atresia intestinal(7).

No detectamos deleción homocigótica o heterocigótica de Fgf10 y Fgfr2b en nuestro paciente.

Se ha descrito también la implicación del gen NOTCH1 con cardiopatía congénita obstructiva izquierda, predominantemente estenosis de la válvula aórtica y válvula aórtica bicúspide, coartación aórtica y corazón izquierdo hipoplásico y la asociación con anomalías vasculares extracardiacas y apple peel, asumiendo la vasculopatía embrionaria la causa de dicho defecto ${ }^{(8)}$. Nuestra paciente presenta como cardiopatía asociada tetralogía de Fallot con doble salida de ventrículo derecho. Tampoco se detectaron variantes en el gen NOTCHI.

En conclusión, la combinación de atresia duodenal con apple peel es excepcional, y aunque está descrita la asociación de anomalías gastrointestinales con la hernia diafragmática congénita, a nuestro entender la combinación de las tres malformaciones no había sido previamente descrita en la literatura científica.

\section{BIBLIOGRAFÍA}

1. Dalla Vecchia LK, Grosfeld JL, West KW, Rescorla FJ, Scherer LR, Engum SA. Intestinal atresia and stenosis: a 25-year experience with 277 cases. Arch Surg. 1998; 133(5): 490-6; discussion 496-7.
2. Tandler J. Zur. Entwickelungsgeschichte des menschlichen duodenum in fruhen Embryonalstadien. Morph Jahrb. 1900; 29: 187216.

3. Louw JH, Barnard CN. Congenital intestinal atresia; observations on its origin. Lancet Lond Engl. 1955; 269(6899): 1065-7.

4. Santulli TV, Blanc WA. Congenital atresia of the intestine: pathogenesis and treatment. Ann Surg. 1961; 154: 939-48.

5. Sweed Y, Puri P. Congenital diaphragmatic hernia: influence of associated malformations on survival. Arch Dis Child. 1993; 69(1 Spec No): 68-70.

6. Castle SL, Naik-Mathuria BJ, Torres MB. Right-sided congenital diaphragmatic hernia, hepatic pulmonary fusion, duodenal atresia, and imperforate anus in an infant. J Pediatr Surg. 2011; 46(7): $1432-4$.

7. Tatekawa Y, Kanehiro H, Nakajima Y. Duodenal atresia associated with 'apple peel' small bowel without deletion of fibroblast growth factor-10 or fibroblast growth factor receptor 2IIIb: report of a case. Surg Today. 2007; 37(5): 430-3.

8. Digilio MC, Magliozzi M, Di Pede A, Valfrè L, Dentici ML, Auriti C, et al. Familial aggregation of 'apple peel' intestinal atresia and cardiac left-sided obstructive lesions: A possible causal relationship with NOTCH1 gene mutations. Am J Med Genet A. 2019; 179(8): 1570-4.

9. Molino JA, García Martínez L, Guillén Burrieza G, Peiró Ibáñez JL, López-Fernández S, Laín A, et al. Outcomes after Split Abdominal Wall Muscle Flap Repair for Large Congenital Diaphragmatic Hernias. Eur J Pediatr Surg Off J Austrian Assoc Pediatr Surg Al Z Kinderchir. 2020; 30(2): 210-4.

10. Zaiss I, Kehl S, Link K, Neff W, Schaible T, Sütterlin M, et al. Associated malformations in congenital diaphragmatic hernia. Am J Perinatol. 2011; 28(3): 211-8.

11. Weber DM, Freeman NV. Duodenojejunal atresia with apple peel configuration of the ileum and absent superior mesenteric artery: observations on pathogenesis. J Pediatr Surg. 1999; 34(9): 1427-9. 
12. Arbell D, Orkin B, Naveh Y, Gur I, Udassin R. Duodenojejunal atresia with absent dorsal mesentery, choledochal cyst, and malrotation in a premature newborn-a case report. J Pediatr Surg. 2006; 41(6): e11-3.

13. Ahmad A, Sarda D, Joshi P, Kothari P. Duodenal atresia with 'applepeel configuration' of the ileum and absent superior mesenteric artery: a rare presentation. Afr J Paediatr Surg AJPS. 2009; 6(2): 120-1.

14. Patil RT, Gupta R, Parelkar SV, Oak S, Sanghvi B, Prakash A, et al. A rare case of duodenal atresia with apple-peel configuration of the small intestine and malrotation. Eur J Pediatr Surg Off J Austrian Assoc Pediatr Surg Al Z Kinderchir. 2011; 21(5): 340-2.

15. Altokhais TI. Duodenal atresia with apple-peel jujenoilial deformity: Case report and review of the literature. J Pediatr Surg Case Rep. 2014; 2(3): 156-8.

16. Alnosair AA, Naga MI, Abdulla MR, Al-Salem AH. Congenital duodenal atresia with 'apple-peel configuration' of the small intestines and absent superior mesenteric artery: A case report and review of literature. J Pediatr Surg Case Rep. 2014; 2(5): 215-8.

17. Pathak M, Narula D. A case of duodenal atresia with apple peel appearance: challenging the current embryology. J Clin Neonatol. 2014; 3(2): 112-4.

18. Saša RV, Ranko L, Snezana C, Lidija B, Djordje S. Duodenal atresia with apple-peel configuration of the ileum and absent superior mesenteric artery. BMC Pediatr. 2016; 16(1): 150.

19. Ben Hamida H, Hadj Salem R, Ben Ameur K, Rassas A, Chioukh FZ, Sakka R, et al. Duodenal Atresia Associated with Apple Peel Atresia and Situs Inversus Abdominus: A Case Report. J Neonatal Surg. 2016; 5(4): 60.

20. Kirtane JM, Bhange SA, Nabi F, Shah V. Duodenal atresia with familial apple peel syndrome: case study with review of literature. BMJ Case Rep. 2019; 12(8): e230160. 